\section{P 275 PATIENTS WHO DIE IN HOSPITAL-IS THERE AN UNMET PALLIATIVE CARE NEED?}

Caroline Bruckner-Holt, ${ }^{1}$ Alice Baker, ${ }^{2}$ Hafiz Aladin, ${ }^{1}$ Lisa Boulstridge ${ }^{1} .{ }^{1}$ Good Hope Hospital, Birmingham, UK; ${ }^{2}$ University of Birmingham

\subsection{6/bmjspcare-2014-000654.316}

Background The majority of people in the UK die in hospital and many more die in hospital than are seen by the inpatient specialist palliative care team (SPCT).

Aims To assess the population of patients who die in hospital and to establish if there is an unmet specialist palliative care need in patients not referred to the SPCT.

Methods We conducted a retrospective case note evaluation and collection of death certificate data of all 109 patients that died at Good Hope Hospital Birmingham in September 2012. We assigned Gold Standards Framework (GSF) prognostic indicator criteria to assess possible palliative care need. We assessed differences between the population of patients referred to the SPCT and those that were not.

Results 19/109 patients were seen by SPCT and 19/109 were known to community SPCT. $48 / 109$ patients died $<72$ hours following admission. 29/109 patients scored 0 on the GSF criteria, with 47/109, 26/109 and 7/109 scoring 1,2 and 3 respectively. Patients referred to the SPCT scored more highly on GSF criteria than those not referred $1.6+/-0.2$ versus 1.0 $+/-0.1, p=0.004$. The time from admission to death was longer in those referred to SPCT 16 days $+/-5.6$ versus 8 days $+/-1.4$ in the group not referred, $p=0.04$.

Conclusions According to assessment of GSF prognostic indicator criteria, nearly $3 / 4$ patients dying within the hospital meet criteria for palliative care need. Those patients that are referred to SPCT score higher so perhaps constitute a group with more comorbidity and thus identification of need is easier. Time to death in the SPCT group was also longer, suggesting those that do not die 'quickly' are identified more readily as having palliative care needs. Increased awareness and education around the importance of assessment of palliative care need amongst clinicians may increase referral to SPCT. 